\title{
AN EVOLUTIONARY CONTINUOUS CASTING PROBLEM OF STEFAN TYPE*
}

\author{
BY \\ JOSÉ-FRANCISCO RODRIGUES \\ University of Lishon, Lishon, Portugal
}

\begin{abstract}
A simplified model for the solidification of an ingot being cast continuously by withdrawing it from a mould at constant rate is studied via the variational inequalities approach. Existence, uniqueness and regularity results are given for this one-phase Stefan type problem, together with a detailed analysis for the free boundary (the solid-liquid interface) particularly with respect to the asymptotic behavior as time $t \uparrow \infty$ where the steady state is attained.
\end{abstract}

1. Introduction. We consider a simplified model of a one-phase Stefan problem with transport representing the solidification of an ingot by the continuous casting process. The portion of ingot taken into account includes the solid-liquid interface $\Phi$ and occupies a cylindrical open bounded domain $\Omega=\Gamma \times] 0$, l[ of $\mathbf{R}^{3}$, where $\Gamma \subset \mathbf{R}^{2}$ is a domain with Lipschitz boundary $\partial \Gamma$ representing the section of the ingot and $l>0$ is the height of the lateral mould. We set $\left.\Gamma_{0}=\Gamma \times\{0\}, \Gamma_{1}=\partial \Gamma \times\right] 0, l\left[\right.$ and $\Gamma_{2}=\Gamma \times\{l\}$; we denote $X=(x, y, z) \in \bar{\Omega}$, the gradient by $\nabla=\left(\partial_{x}, \partial_{y}, \partial_{z}\right)$, so $\Delta=\nabla \cdot \nabla$, the time derivative by $\partial_{t}$ and we set $\left.Q_{T}=\Omega \times\right] 0, T\left[, \Gamma_{T}=\Gamma \times\right] 0, T[$ for any $0<T<\infty$.

If the free boundary at each instant is given by $\Phi(t)=\{X \in \Omega: z=\phi(x, y, t)\}$ for a smooth function $\phi: \Gamma \times[0, T[\mapsto[0, l[$ and if we consider the temperature $\theta=\theta(X, t)$ renormalized such that $\theta=0$ in the liquid part and $\theta>0$ in the solid one, the classical formulation of the continuous casting problem in a fixed frame and with casting velocity given by $\vec{V}=b \vec{z}$ (for a given constant $b>0$ ) can be stated as follows (see [ $\mathbf{B r}]$ and [R4] for more details).

Problem (C). Find two smooth functions $\theta=\theta(x, y, z, t) \geqslant 0$ and $\phi=\phi(x, y, t)>0$ defined in $\bar{\Omega} \times[0, T]$ and $\Gamma \times[0, T]$, respectively, such that

$$
\begin{gathered}
\partial_{t} \theta-\Delta \theta+b \partial_{z} \theta=0 \quad \text { if }(X, t): z>\phi(x, y, t) ; \\
\theta=0 \quad \text { if }(X, t): z \leqslant \phi(x, y, t) ; \\
\partial_{z} \theta-\partial_{x} \theta \partial_{x} \phi-\partial_{y} \theta \partial_{y} \phi=\lambda\left(b-\partial_{t} \phi\right) \quad \text { if }(X, t): z=\phi(x, y, t) ;
\end{gathered}
$$

${ }^{*}$ Received April 22, 1985. 


$$
\begin{gathered}
\theta=0 \text { on } \Gamma_{0} \text { and } \theta=h(x, y, t) \text { on } \Gamma_{2} \text { for } t>0 ; \\
-\partial \theta / \partial n=\alpha(\theta-\rho) \quad \text { on } \Gamma_{1} \text { for } t>0 ; \\
\theta(X, 0)=\theta_{0}(X) \text { for } X \in \Omega \text { at } t=0 .
\end{gathered}
$$

Here $\lambda$ and $\alpha$ are positive constants denoting respectively the latent heat and the cooling coefficient, $h>0, \rho \geqslant 0$ and $\theta_{0} \geqslant 0$ are given functions representing known temperatures, compatible with the one-phase model in a sense to be made precise. By the maximum principle, from (1.1) one concludes that it must be $\theta>0$ for $z>\phi(x, y, t)$. We remark that in (1.3) the Stefan condition at the free boundary establishes the balance between the flux of heat and the velocity of propagation of the interface in the ingot; also from the maximum principle one finds that $\partial_{t} \phi \leqslant b$, which is physically admissible.

There is an important analogy between this model and the free boundary problem representing the nonsteady filtration of a compressible fluid through a rectangular porous dam (see [T3]), which is a quite different physical phenomenon. Therefore our mathematical treatment will follow similar techniques, but since the boundary conditions are essentially different we have a new problem with interesting features as was already observed in [Br] and [R4]. Of course one could also consider a two-dimensional model in this framework.

The formulation of Problem (C) contains, in particular, the formulation of the stationary case in which $\theta$ and $\phi$ are time-independent.

In Section 2 we transform Problem (C) and we consider the parabolic variational inequality approach with nonclassical boundary conditions, according to $[\mathrm{Br}]$, where a combination of the method of Baiocchi for the dam problem [BC], with its extension by Duvaut to the one-phase Stefan problem [D], was introduced in a way similar to the works of Torelli $[\mathbf{T 1}, 2,3]$. We derive a new existence theorem and we obtain several properties which are in fact analogous to the evolution dam problem.

In Section 3, we study the steady-state problem, namely the regularity of the solution and, by means of comparison arguments, we discuss the existence of the free boundary and the equivalence between the initial problem (C) and its transformed problem. It turns out that for small velocities $b$ and a large lateral cooling near $\Gamma_{0}$ there is no free boundary and Problem $(\mathrm{C})$ is consequently ill-posed. Nevertheless, one can assure its existence by imposing natural compatibility assumptions on the physical data. In this case we can give a new stability result for the free boundary which, in particular, is important for the study of its asymptotic behavior as $t \rightarrow \infty$. We extend the comparison and stability results to the evolutionary case in Section 4 . In particular, these properties provide sufficient conditions to the analysis of the free boundary in Section 5, namely the local $C^{1}$ regularity for $\phi(x, t)$, as in the compressible dam problem $[\mathbf{K}]$, and the equivalence between Problem (C) and its variational inequality formulation.

In the last part of this paper we study the asymptotic behavior as $t \rightarrow \infty$, and in Section 6 we state several results on the order of convergence for the variational solutions and for the free boundaries, respectively in $L^{2}(\Omega), H^{1}(\Omega) \cap H_{\mathrm{loc}}^{2}(\Omega)$ and in $L^{1}(\Gamma)$. Finally, in Section 7, we establish the strong stabilization of the free boundary, locally in Hölder spaces together with its rate of convergence. 
2. The variational inequality approach. The condition (1.3) induces a discontinuity of $\nabla \theta$ across the free boundary and it can be formally condensed, together with (1.1) and (1.2) into a single equation in the sense of the distributions $\mathscr{D}^{\prime}\left(Q_{T}\right)$.

Proposition 2.1. Let $\chi^{+}=\chi^{+}(\theta)$ denote the characteristic function of the set

$$
Q^{+}=\left\{(X, t) \in Q_{T}: z>\phi(x, y, t)\right\}=\{\theta(X, t)>0\} .
$$

If $\theta$ and $\phi$ are smooth (say $\phi \in C^{1}\left(\Gamma_{T}\right), \theta \in C^{0}\left(Q_{T}\right)$ with $\nabla \theta \in C^{1}\left(Q^{+} \cup \Phi\right)$ ), then (1.1, $2,3)$ are equivalent to

$$
\partial_{t} \theta-\Delta \theta+b \partial_{z} \theta=-\lambda\left(\partial_{t}+b \partial_{z}\right) \chi^{+}(\theta) \text { in } \mathscr{D}^{\prime}\left(Q_{T}\right) .
$$

Proof. Let $\Phi=\bigcup_{0<t<T} \Phi(t)$ and $\vec{\nu}=l\left(\partial_{x} \phi, \partial_{y} \phi,-1, \partial_{t} \phi\right)$ be the outward normal to $\Phi \cap \partial Q^{+}$, with $l^{-2}=\left(\partial_{x} \phi\right)^{2}+\left(\partial_{y} \phi\right)^{2}+1+\left(\partial_{t} \phi\right)^{2}$. For any $\psi \in \mathscr{D}\left(Q_{T}\right)$ and remarking that

$$
\begin{gathered}
\left\langle\left(\partial_{t}+b \partial_{z}\right) \chi^{+}, \psi\right\rangle_{Q_{T}}=-\int_{Q^{+}}\left(\partial_{t}+b \partial_{z}\right) \psi=-\int_{\Phi} \psi\left(\partial_{t} \phi-b\right) l, \\
\langle\Delta \theta, \psi\rangle_{Q_{T}}=\int_{Q^{+}} \theta \Delta \psi=-\int_{Q^{+}} \nabla \theta \cdot \nabla \psi \\
=\int_{Q^{+}} \psi \Delta \theta-\int_{\Phi} \psi\left(\partial_{x} \theta \partial_{x} \phi+\partial_{y} \theta \partial_{y} \phi-\partial_{z} \theta\right) l
\end{gathered}
$$

one easily concludes that $(1.1,2,3)$ are equivalent to

$$
\left\langle\partial_{t} \theta-\Delta \theta+b \partial_{z} \theta, \psi\right\rangle_{Q_{T}}=-\lambda\left\langle\left(\partial_{t}+b \partial_{z}\right) \chi^{+}, \psi\right\rangle_{Q_{T}} .
$$

In order to "regularize" the right side of (2.1) we transform the problem by introducing the new function $[\mathbf{B r}]$

$$
u(X, t)=b \int_{0}^{t} \theta(x, y, z+b(\tau-t), \tau) d \tau, \quad X \in \bar{\Omega}, t \geqslant 0,
$$

being $\theta=\theta(X, t)$ extended by zero for $-\infty<z \leqslant \phi(x, y, t)$. In an equivalent way one can write (2.2) in the form

$$
u(X, t)=\int_{(z-b t)^{+}}^{z} \theta\left(x, y, \zeta, t+\frac{\zeta-z}{b}\right) d \zeta, \quad X \in \bar{\Omega}, t \geqslant 0,
$$

where $v^{+}=\max (v, 0)$. Remark that, if $\theta$ is time-independent, for $t \geqslant l / b$ one obtains the Baiocchi transformation (see $[\mathbf{B C}]$ ). The inverse transformation is given by the following proposition.

Proposition 2.2. If $\theta \in L_{\text {loc }}^{1}\left(Q_{T}\right)$, one has

$$
\partial_{t} u / b+\partial_{z} u=\theta, \text { a.e. in } Q_{T} .
$$


Proof. Let $\psi \in \mathscr{D}\left(Q_{T}\right)$ and recall that $\left.Q_{T}=\Gamma \times\right] 0, l[\times] 0, T[$. Denoting $\tilde{\psi}(x, y, \zeta, \tau, t)$ $=\psi(x, y, \zeta-b(\tau-t), t)$ one has

$$
\begin{aligned}
\left\langle u, \partial_{t} \psi / b-\partial_{z} \psi\right\rangle & =\int_{0}^{T} d t \int_{\Gamma} d x d y \int_{0}^{t} d \tau \int_{0}^{t} \theta(x, y, z+b(\tau-t), \tau)\left[\partial_{t} \psi-b \partial_{z} \psi\right](X, t) d z \\
& =\int_{0}^{T} d t \int_{\Gamma} d x d y \int_{0}^{t} d \tau \int_{b(\tau-t)}^{l+b(\tau-t)} \theta(x, y, \zeta, t) \partial_{t} \tilde{\psi}(x, y, \zeta, \tau, t) d \zeta \\
& =\int_{0}^{T} d \tau \int_{\Gamma} d x d y \int_{0}^{l} d \zeta \theta(x, y, \zeta, t) \int_{\tau}^{T} \partial_{t} \tilde{\psi}(x, y, \zeta, \tau, t) d t \\
& =-\int_{Q_{T}} \theta \psi d X d t
\end{aligned}
$$

since $\psi=0$ for $\zeta \leqslant b(\tau-t)$ and for $\zeta \geqslant l+b(\tau-t), 0 \leqslant \tau<t \leqslant T$.

Assume that the initial position of the free boundary, which is determined by the initial temperature $\theta_{0}$, is given by a positive continuous function $\phi_{0}$, so that $\Phi(0): z=\phi_{0}(x, y)$. Set

$$
\mathcal{O}=\left\{(X, t) \in Q_{T}: z>\phi_{0}(x, y)+b t\right\}
$$

and remark that for $t \geqslant l / b$ one has $\mathcal{O} \cap\{\tau=t\}=\varnothing$ and, since in Problem (C) one must have $\partial_{t} \phi \leqslant b, \mathcal{O} \subset Q^{+}$also.

Denoting by $\chi_{\mathcal{O}}=\chi_{\mathcal{O}}(X, t)$ the characteristic function of $\mathcal{O}$, we introduce the following functions

$$
\begin{aligned}
& f(X, t)=b \theta_{0}(x, y, z-b t) \chi_{\mathcal{O}}-\lambda b\left[1-\chi_{\mathcal{O}}\right], \quad X \in \Omega, t \geqslant 0, \\
& g(X, t)=\int_{(z-b t)^{+}}^{z} \rho\left(x, y, \zeta, t+\frac{\zeta-z}{b}\right) d \zeta, \quad X \in \Gamma_{1}, t>0 .
\end{aligned}
$$

Proposition 2.3. If $\theta$ is a solution to Problem (C) with $\phi(x, y, t)>0$ for $(x, y) \in \Gamma$, $t \geqslant 0$, then the transformation (2.2) implies

$$
\begin{gathered}
u(X, t) \geqslant 0, \quad X \in \Omega, t \geqslant 0 ; \quad Q^{+}=\left\{(X, t) \in Q_{T}: u(X, t)>0\right\} ; \\
\partial_{t} u-\Delta u+b \partial_{z} u=f \chi_{Q^{+}} \quad \text { a.e. in } Q_{T} \\
u=0 \quad \text { on } \Gamma_{0}, t>0 \\
-\partial u / \partial n=\alpha(u-g) \quad \text { on } \Gamma_{1}, t>0 ; \\
\partial_{t} u / b+\partial_{z} u=h \quad \text { on } \Gamma_{2}, t>0 ; \\
u(X, 0)=0, \quad X \in \Omega .
\end{gathered}
$$

Proof. From the preceding propositions the only condition which requires an additional argument is (2.9). We distinguish between $\mathcal{O}$ and $Q_{T} \backslash \mathcal{O}$. 
Since $\mathcal{O} \subset Q^{+}$, for $(X, t) \in \mathcal{O}$ one has

$$
\begin{aligned}
\Delta u(X, t) & =b \int_{0}^{t} \Delta \theta(x, y, z+b(\tau-t), \tau) d \tau \\
& =b \int_{0}^{t}\left[\partial_{t} \theta+b \partial_{z} \theta\right](x, y, z+b(\tau-t), \tau) d \tau \\
& =b[\theta(x, y, z+b(\tau-t), \tau)]_{\tau=0}^{\tau=t}=b \theta(X, t)-b \theta_{0}(x, y, z-b t) \\
& =\partial_{t} u+b \partial_{z} u-b \theta_{0}(x, y, z-b t) .
\end{aligned}
$$

On the other hand, from (2.1) and (2.4) one has

$$
\left(\partial_{t}+b \partial_{z}\right)\left(\partial_{t} u-\Delta u+b \partial_{z} u+\lambda b \chi_{Q^{+}}\right)=0
$$

which implies that $\partial_{t} u-\Delta u+b \partial_{z} u+\lambda b \chi_{Q^{+}}$is equal to some constant function along the straight lines $z-b t=C$; since, by assumption, $u=\chi_{Q^{+}}=0$ for $0<z<\phi(x, y, t)$, it follows that (2.9) holds in $Q_{T} \backslash \mathcal{O}$.

Now, integrating by parts, it is classical to conclude that $u$ verifying the conditions (2.8)-(2.13) should be the solution to the following parabolic variational inequality for a.e. $t>0$ :

$$
\begin{gathered}
u(t) \in \mathbf{K}, \quad u(0)=0 ; \\
\left(\partial_{t} u(t), v-u(t)\right)+a(u(t), v-u(t)) \geqslant\langle L(t), v-u(t)\rangle, \quad \forall v \in \mathbf{K}
\end{gathered}
$$

where we have introduced the following notations:

$$
\begin{gathered}
V=\left\{v \in H^{1}(\Omega): v=0 \text { on } \Gamma_{0}\right\} ; \quad \mathbf{K}=\{v \in V: v \geqslant 0 \text { in } \Omega\} ; \\
a(u, v)=\int_{\Omega} \nabla u \cdot \nabla v+b \int_{\Omega} \partial_{z} u v+\alpha \int_{\Gamma_{1}} u v \quad(u, v \in V) ; \\
(u, v)=\int_{\Omega} u v+\frac{1}{b} \int_{\Gamma_{2}} u v \quad(u, v \in V) ; \\
\langle L(t), v\rangle=\int_{\Omega} f(t) v+\alpha \int_{\Gamma_{1}} g(t) v+\int_{\Gamma_{2}} h(t) v \quad(v \in V) .
\end{gathered}
$$

We observe that the nonclassical boundary condition (2.12) has been included in (2.18), but it requires the additional regularity $\partial_{t} u(t) \in V$ for a.e. $t>0$. Therefore we must consider the existence of strong solutions to (2.14) and (2.15) in the abstract framework $V \subset H \subset V^{\prime}$ (see [L1]) where $H$ is the Hilbert space obtained from $V$ with the inner product (2.18).

LEMMA 2.1. We have the following isomorphism

$$
H=\bar{V}^{(\cdot \cdot)} \simeq\left[L^{2}(\Omega) \times L^{2}\left(\Gamma_{2}\right)\right] .
$$

Proof. By continuity of the trace operator one has $H \subset\left[L^{2}(\Omega) \times L^{2}\left(\Gamma_{2}\right)\right]$ and it is sufficient to show that its orthogonal $H^{\perp}$ reduces to $\{0,0\}$. If $\left(v_{1}, v_{2}\right) \in H^{\perp}$, one has

$$
\int_{\Omega} v_{1} w_{1}+\frac{1}{b} \int_{\Gamma_{2}} v_{2} w_{2}=0, \quad \text { for all } w \simeq\left\{w_{1}, w_{2}\right\} \in H
$$


taking $w \in \mathscr{D}(\Omega) \subset H$, one finds first $v_{1}=0$; afterwards with an arbitrary $w \in V \subset H$ one concludes $v_{2}=0$.

Next we make the following assumptions on the data in order to apply the existence and regularity theory for abstract variational inequalities of evolution:

$$
\begin{gathered}
\phi_{0} \in C^{0}(\Gamma), \quad \phi_{0}(x, y)>0 \text { for all }(x, y) \in \Gamma ; \\
\theta_{0} \in C^{0}(\bar{\Omega}), \quad \partial_{z} \theta_{0} \in L^{\infty}(\Omega) \text { with }\left\{\theta_{0}>0\right\}=\left\{z>\phi_{0}(x, y)\right\} ; \\
h \in H^{1}\left(0, T ; L^{2}\left(\Gamma_{2}\right)\right), \quad h>0 ; \\
\rho \in H^{1}\left(0, T ; L^{2}\left(\Gamma_{1}\right)\right), \quad \rho \geqslant 0 .
\end{gathered}
$$

THEOREM 2.1. Under assumptions (2.20)-(2.23) there exists a unique solution to the variational inequality (2.14), (2.15), verifying

$$
u \in H^{1}(0, T ; V) \cap W^{1, \infty}(0, T ; H) \cap W_{p}^{2,1}\left(Q^{\prime}\right) \cap C^{0}\left(\left(\Omega \cup \Gamma_{0}\right) \times[0, T]\right)
$$

for any $p<\infty$ with $\left.Q^{\prime}=\Omega^{\prime} \times\right] 0, T\left[\right.$ for any open set $\Omega^{\prime}$ such that $\bar{\Omega}^{\prime} \subset \Omega \cup \Gamma_{0}$. Furthermore, $u$ verifies the equation

$$
\partial_{t} u-\Delta u+b \partial_{z} u=f \chi_{\{u>0\}} \quad \text { a.e. in } Q_{T} .
$$

Proof. The first part follows immediately from the general theory (see [L1]) provided one assures $L(0) \in H$ and $L \in H^{1}\left(0, T ; V^{\prime}\right)$. Since $h(0) \in L^{2}\left(\Gamma_{2}\right)$ and for any $v \in V$ one has

$$
\langle L(0), v\rangle=\int_{\Omega} b \theta_{0}(X) v-\lambda b \int_{\Omega}\left[1-\chi_{\mathcal{O}}(0)\right] v+\int_{\Gamma_{2}} h(0) v=(L(0), v),
$$

by density one concludes $L(0) \in H$. The other condition follows at once from the assumptions, which imply $\partial_{t} f \in L^{2}\left(0, T ; V^{\prime}\right)$ (see Lemma 2.2 below).

Since $f \in L^{\infty}\left(Q_{T}\right)$, the local regularity is standard from the corresponding results for parabolic equations [LSU] and its extension to variational inequalities [L2]. Then $W_{p}^{2.1}\left(Q^{\prime}\right) \subset C^{0}\left(\bar{Q}^{\prime}\right)$ for $p>(3+2) / 2$ and (2.24) follows. Finally, in the open set $\left\{(X, t) \in Q_{T}: u(X, t)>0\right\}$ the equation $\partial_{t} u-\Delta u+b \partial_{z} u=f$ is verified and in its complementary set $\{u=0\}$ one has $\partial_{t} u=\Delta u=\partial_{z} u=0$ a.e.

LEMMA 2.2. Under assumptions (2.20) and (2.21) one has

$$
\begin{gathered}
\partial_{t} f, \partial_{t} \chi_{\mathcal{O}} \in L^{\infty}\left(0, \infty ; V^{\prime}\right) \cap L^{\infty}\left(0, \infty ; W^{-1, p}(\Omega)\right), \quad \forall p<\infty . \\
\left(\partial_{t}+b \partial_{z}\right) f=\left(\partial_{t}+b \partial_{z}\right) \chi_{\mathcal{O}}=0 .
\end{gathered}
$$

Moreover, if we also assume

$$
\partial_{z} \theta_{0} \geqslant 0 \quad \text { a.e. in } \Omega
$$

we have

$$
\partial_{t} f \leqslant 0 \quad \text { and } \quad \partial_{z} f \geqslant 0 \text { on } Q_{T} .
$$

Proof. Since $\chi_{\mathcal{O}}(t)=0$ for $t \geqslant l / b$ we can limit ourselves to the finite interval ]0,T[, with $l / b<T<\infty$. From the definition of $f$ one considers the terms $f_{0}=\theta_{0}(x, y, z-$ $b t) \chi_{\mathcal{O}}(X, t)$ and $\chi_{\mathcal{O}}$. Recaliing that $f_{0} \in L^{\infty}\left(0, T ; L^{\infty}(\Omega)\right) \subset \mathscr{D}^{\prime}\left(0, T ; V^{\prime}\right)=$ 
$\mathscr{L}\left(\mathscr{D}(0, T), V^{\prime}\right)$, its derivative is given by

$$
\partial_{t} f_{0}(\psi)=-\int_{0}^{T} f_{0}(t) \psi^{\prime}(t) d t, \quad \forall \psi \in \mathscr{D}(0, T) .
$$

Therefore, in the duality $\left\langle V^{\prime}, V\right\rangle$ one has, for $v \in V$

$$
\begin{aligned}
& \left\langle\partial_{t} f_{0}(\psi), v\right\rangle=-\left\langle\int_{0}^{T} f_{0}(t) \psi^{\prime}(t) d t, v\right\rangle=-\int_{\Gamma} d x d y \int_{0}^{T} \psi^{\prime}(t)\left\{\int_{\phi_{0}+b t}^{l} \theta_{0}(x, y, z-b t) v d z\right\} \\
& \quad=-\int_{\Omega} v\left(\int_{0}^{\left(z-\phi_{0}\right) / b}\left\{\partial_{t}\left(\theta_{0}(x, y, z-b t) \psi(t)\right)+b \partial_{z} \theta_{0}(x, y, z-b t) \psi(t)\right\} d t\right) d X \\
& \quad=-\int_{\Gamma} d x d y \int_{0}^{T} \psi(t)\left\{\int_{\phi_{0}+b t}^{l} b \partial_{z} \theta_{0}(x, y, z-b t) v d z\right\} d t \\
& \quad=-\int_{0}^{T} \psi(t)\left[\int_{\Omega} b \chi_{\mathcal{O}}(t) \partial_{z} \theta_{0}(x, y, z-b t) v d X\right] d t,
\end{aligned}
$$

and analogously

$$
\begin{aligned}
\left\langle\partial_{t} \chi_{\mathcal{O}}, v\right\rangle & =-\int_{\Omega} v\left(\int_{0}^{\left(z-\phi_{0}\right) / h} \psi^{\prime}(t) d t\right) d X=-\int_{\Gamma} d x d y \int_{0}^{l} v \psi\left(\frac{z-\phi_{0}}{b}\right) d z \\
& =-\int_{\Gamma} d x d y \int_{-\phi_{0} / b}^{\left(l-\phi_{0}\right) / b} \psi(t) v\left(x, y, b t+\phi_{0}\right) b d t \\
& =-\int_{\Gamma} d x d y \int_{0}^{\left(l-\phi_{0}\right) / b} \psi(t)\left\{v(x, y, H)-\int_{b t+\phi_{0}}^{l} \partial_{z} v(x, y, z) d z\right\} b d t \\
& =\int_{0}^{T} \psi(t)\left\{b \int_{\Omega} \chi_{\mathcal{O}}(t) \partial_{z} v\right\} d t-\int_{0}^{T} \psi(t)\left\{b \int_{\Gamma_{2}} \chi_{\mathcal{O}}(t) v\right\} d t .
\end{aligned}
$$

Whence one concludes that $\partial_{t} f_{0}$ and $\partial_{t} \chi_{0}$ can be identified with the applications that for each $t \in] 0, T$ [ map into the elements of $V^{\prime}$ given, respectively, by

$$
\partial_{t} f_{0}(t): v \mapsto-b \int_{\Omega} \chi_{0}(t) \partial_{z} \theta_{0}(x, y, z-b t) v,
$$

and

$$
\partial_{t} \chi_{\mathcal{O}}(t): v \mapsto b \int_{\Omega} \chi_{\mathcal{O}}(t) \partial_{z} v-b \int_{\Gamma_{2}} \chi_{\mathcal{O}}(t) v,
$$

from which (2.26) follows. Taking $v \in W_{0}^{1, p^{\prime}}(\Omega)$ and arguing with the duality $\left\langle W^{-1, p}(\Omega)\right.$, $\left.W_{0}^{1, p^{\prime}}(\Omega)\right\rangle$ one concludes the last assertion of (2.26).

To prove (2.27) we approximate $\chi_{\mathcal{O}}$ by smooth functions $\chi_{\varepsilon}(X, t)=q_{\varepsilon}(x, y, z-b t)$, which verify $\left(\partial_{t}+b \partial_{z}\right) \chi_{\varepsilon}=0$. Since $\left(\partial_{t}+b \partial_{z}\right) \theta(x, y, z-b t)=0$ also, (2.27) follows easily.

Recalling that $\chi_{\mathcal{O}}=1$ if $z>\phi_{0}(x, y)+b t$ and $\chi_{\mathcal{O}}=0$ if $z \leqslant \phi_{0}(x, y)+b t$, one has that $\chi_{\mathcal{O}}(X, t) \geqslant \chi_{\mathcal{O}}(X, t+\tau), \forall \tau>0$ (resp. $\left.\chi_{\mathcal{O}}(x, y, z, t) \leqslant \chi_{\mathcal{O}}(x, y, z+\tau, t)\right)$ implies $\partial_{t} \chi_{\mathcal{O}} \leqslant 0$ (resp. $\partial_{z} \chi_{\mathcal{O}} \geqslant 0$ ) from which (2.29) follows easily provided that (2.28) holds. 
3. The steady-state problem. If we assume the free boundary stabilized with respect to the mould and the temperature time-independent, we have an elliptic problem whose classical formulation is given by (1.1)-(1.6) where all the references to $t$ are neglected. As we have already remarked, the Baiocchi transformation (2.3) now takes the form

$$
u_{\infty}(X)=\int_{0}^{z} \theta_{\infty}(x, y, \zeta) d \zeta, \quad(x, y, z) \in \bar{\Omega},
$$

(the subscript " $\infty$ " stands for the steady-state functions) and we obtain the following elliptic variational inequality $[\mathbf{B r}]$ :

$$
u_{\infty} \in \mathbf{K} ; \quad a\left(u_{\infty}, v-u_{\infty}\right) \geqslant\left\langle L_{\infty}, v-u_{\infty}\right\rangle, \quad \forall v \in \mathbf{K}
$$

with the preceding notations and

$$
\left\langle L_{\infty}, v\right\rangle=-\lambda b \int_{\Omega} v+\alpha \int_{\Gamma_{1}} g_{\infty} v+\int_{\Gamma_{2}} h_{\infty} v, \quad v \in V
$$

(here $h_{\infty}=h_{\infty}(x, y)>0$ is given and $g_{\infty}(z)=\int_{0}^{z} \rho_{\infty}(\zeta) d \zeta$, for a given cooling temperature $\left.\rho_{\infty}=\rho_{\infty}(z) \geqslant 0\right)$. One has the following results.

THEOREM 3.1. Let $\rho_{\infty} \in L^{\infty}(0, l), h_{\infty} \in C^{0.1}\left(\bar{\Gamma}_{2}\right)$ be compatible in the sense that $-\partial h_{\infty} / \partial n=\alpha\left(h_{\infty}-\rho_{\infty}\right)$ on $\partial \Gamma_{2}$, which we assume of class $C^{2, \beta}$. Then the unique solution to (3.2) is such that

$$
u_{\infty} \in W^{2, p}(\Omega) \cap C^{1, \gamma}(\bar{\Omega}) \cap W_{\mathrm{loc}}^{2, \infty}(\Omega), \quad \forall 1 \leqslant p<\infty, \forall 0 \leqslant \gamma<1 .
$$

Moreover, we have $\theta_{\infty}=\partial_{z} u_{\infty} \geqslant 0$ in $\bar{\Omega}$ and the solid region is given by a supergraph $\left\{z>\phi_{\infty}(x, y)\right\}=\left\{\theta_{\infty}>0\right\}=\left\{u_{\infty}>0\right\}$ and the free boundary, given by

$$
\Phi_{\infty}=\partial\left\{u_{\infty}>0\right\} \cap \Omega=\left\{X=(x, y, z): z=\phi_{\infty}(x, y)\right\},
$$

is an analytic surface whenever $\phi_{\infty}>0$. In this case, $\left(\theta_{\infty}, \phi_{\infty}\right)$ is the classical solution to the steady-state continuous casting problem.

Proof. The existence, uniqueness and local $W_{\text {loc }}^{2, x}$-regularity for the solution follows from the general theory (see $[\mathbf{B C}],[\mathbf{K S}]$ or $[\mathbf{F}])$. The regularity up to the boundary was proved in $[\mathbf{R 1}]$ and it is based on the compatibility condition and the analogous regularity for the corresponding linear mixed boundary-value problem. The relation between the solution $u_{\infty}$ and the temperature $\theta_{\infty}$ requires a further analysis and it has been discussed in [R1] (see also [CR]). If $\phi_{\infty}>0$ (we shall see below sufficient conditions to have this property) we can apply the theory developed for the analysis of the free boundary in the Baiocchi dam problem (see $[\mathbf{B C}],[\mathbf{K S}]$ or $[\mathbf{F}]$ ) to get the conclusions (see $[\mathbf{R 1}]$ ), in particular to conclude the existence of the classical solution $\theta_{\infty}$.

Remark 3.1. The $C^{2, \beta}$ regularity for $\partial \Gamma$ is only necessary in order to assume the global $W^{2, p} \cap C^{1, \alpha}$ smoothness for the variational solution $u_{\infty}$. The second part of Theorem 3.1, being essentially local in nature, still holds for any $\partial \Gamma$ Lipschitz continuous.

One advantage of the variational approach is the possibility of obtaining easily quite natural monotonicity results for the solution and for the free boundary, together wth some a priori bounds, by the well-known techniques of the weak maximum principle. 
Proposition 3.1. If $u$ and $\hat{u}$ denote the solutions to (3.2) with data $b, h, g$ and $\hat{b}, \hat{h}, \hat{g}$, respectively, then $b \geqslant \hat{b}, g \leqslant \hat{g}$, and $h \leqslant \hat{h}$ imply $u \leqslant \hat{u}$ and $\phi \geqslant \hat{\phi}$. Furthermore, if $\hat{\phi}>0, \theta \leqslant \hat{\theta}$ also.

Proof. Set $w=u-\hat{u}$ and take $v=u-w^{+} \in \mathbf{K}$ (resp. $v=\hat{u}+w^{+} \in \mathbf{K}$ ) in the variational inequality for $u$ (resp. $\hat{u}$ ). We have, taking the hypothesis into account,

$$
\begin{aligned}
& \int_{\Omega} \nabla w \cdot \nabla\left(w^{+}\right)+b \int_{\Omega} \partial_{z} w w^{+}+\alpha \int_{\Gamma_{1}}\left|w^{+}\right|^{2}+(b-\hat{b}) \int_{\Omega} \partial_{z} \hat{u} w^{+} \\
& \leqslant-\lambda(b-\hat{b}) \int_{\Omega} w^{+} \leqslant 0 .
\end{aligned}
$$

Recalling that $\partial_{z} \hat{u} \geqslant 0$ and since

$$
\int_{\Omega} \partial_{z} w w^{+}=\frac{1}{2} \int_{\Omega} \partial_{z}\left(w^{+}\right)^{2}=\frac{1}{2} \int_{\Gamma_{2}}\left(w^{+}\right)^{2} \geqslant 0
$$

one obtains

$$
\int_{\Omega}\left|\nabla\left(w^{+}\right)\right|^{2}=0
$$

which, together with $w=0$ on $\Gamma_{0}$ implies $u-\hat{u}=w \leqslant 0$. Then $\phi \geqslant \hat{\phi}$ follows immediately. Finally, we conclude $\theta \leqslant \hat{\theta}$ by applying the classical maximum principle in the open set $\{\theta>0\}$.

In order to get an a priori frame for the free boundary, let us assume

$$
0<m \leqslant h_{\infty} \leqslant M \leqslant \rho_{\infty}(l) \text { and } \rho_{\infty}^{\prime} \leqslant b\left(\lambda+\rho_{\infty}\right) \text { for } 0 \leqslant z \leqslant l .
$$

Set $N=l-m /[b(\lambda+M)]$ and define

$$
\sigma=\sigma(z)=\left\{\begin{array}{l}
0 \quad \text { if } 0 \leqslant z \leqslant N^{+} \\
b(\lambda+M)\left(z-N^{+}\right)^{2} / 2 \quad \text { if } N^{+} \leqslant z \leqslant l .
\end{array}\right.
$$

Proposition 3.2. Assuming (3.6) one has

$$
0 \leqslant \sigma \leqslant u_{\infty} \leqslant g_{\infty} \text { in } \bar{\Omega} .
$$

Consequently, if $\rho_{\infty}=0$ for $0<z<d<l$ one has a lower bound for the free boundary given by $\phi_{\infty} \geqslant d>0$. On the other hand, the upper bound $0 \leqslant \phi_{\infty} \leqslant N^{+}$implies the nonexistence of the free boundary if $N^{+}=0$, i.e., if $0<b \leqslant m /[l(\lambda+M)]$.

Proof. Take $v=u_{\infty}+w \in \mathbf{K}$ in (3.2) for $w=\left(u_{\infty}-g_{\infty}\right)^{+}$and $w=\left(\sigma-u_{\infty}\right)^{+}$. In the first case we get

$$
\int_{\Omega}|\nabla w|^{2}+b \int_{\Omega} \partial_{z} w w+\alpha \int_{\Gamma_{1}} w^{2} \leqslant \int_{\Omega}\left(\rho_{\infty}^{\prime}-b \rho_{\infty}-\lambda b\right) w+\int_{\Gamma_{2}}\left(h_{\infty}-\rho_{\infty}(l)\right) w \leqslant 0
$$

from which we obtain $u_{\infty} \leqslant g_{\infty}$, and in the second one

$$
\int_{\Omega}|\nabla w|^{2}+b \int_{\Omega} \partial_{z} w w+\alpha \int_{\Gamma_{1}}\left(g_{\infty}-u_{\infty}\right) w \leqslant Y
$$

with

$$
Y=\int_{\left\{z \geqslant N^{+}\right\}} b\left\{b(\lambda+M)\left(z-N^{+}\right)-M\right\} w+\int_{\Gamma_{2}}\left\{(b(\lambda+M))\left(l-N^{+}\right)-h_{\infty}\right\} w .
$$


From assumptions (3.6) one finds $Y \leqslant 0$ and, using the first part, one concludes $u_{\infty} \geqslant \sigma$, proving our statements (note that $\rho_{\infty}=0$ for $0<z<d$ implies also $g_{\infty}=0$ there).

Remark 3.2. These types of comparison results have been already considered in $[\mathbf{B r}]$ in the particular case of $h_{\infty}=\rho_{\infty} \equiv 1$ and with respect to the coefficient $\alpha$ : if $\alpha \leqslant \hat{\alpha}$ then $u \leqslant \hat{u}$. One could also prove that $\lambda \geqslant \hat{\lambda}$ implies $u \leqslant \hat{u}$, which is still a natural property from the physical point of view. However, the possibility of nonexistence of the free boundary for the solution of the variational inequality raises the question when the problems for $\theta_{\infty}$ and for $u_{\infty}$ are equivalent. It turns out that for data verifying (3.6) the Problem (C) may be ill-posed for small velocities $b \leqslant m /[l(\lambda+M)]$. Nevertheless, one is able to show that for $b$ large the free boundary always appears, as well as under additional assumptions on the cooling temperature at $z=0$.

TheOREM 3.2. Under conditions (3.6), for any $(x, y) \in \Gamma$ there exists $b^{*}=b^{*}(x, y)$ such that $\phi^{b}(x, y)>0$ for every $b \geqslant b^{*}$, where $\phi^{b}$ denotes the free boundary for the velocity $b$. Furthermore, if

$$
\rho_{\infty}(0)=0 \text { and } \rho_{\infty}^{\prime}(0)<\lambda b \text {, for any fixed } b>0,
$$

then we have $\partial_{z} u_{\infty}=0$ on $\Gamma_{0}$ and $\phi_{\infty}(x, y)>0$ for any $(x, y) \in \Gamma$.

Proof. The first part has been shown in [R1] by constructing a supersolution $\eta$ to (3.2) (i.e., $\eta \geqslant g_{\infty}$ on $\partial \Omega$ and $-\Delta \eta+b \partial_{z} \eta \geqslant-\lambda b$ in $\Omega$ ) such that $\eta$ vanishes in a small neighborhood of $(x, y) \in \Gamma$ in $\Omega$.

For the last part, introduce $\tilde{\Omega}=\Gamma \times]-l, l\left[, \tilde{\Gamma}_{1}=\partial \Gamma \times\right]-l, l\left[\right.$ and $\tilde{\Gamma}_{0}=\Gamma \times\{-l\}$ and let $\tilde{u}$ be the solution in $H^{1}(\tilde{\Omega})$ of the variational inequality analogous to (3.2) with $\tilde{a}(\cdot, \cdot), \tilde{\mathbf{K}}$, and $\tilde{L}_{\infty}$ defined analogously with $\tilde{g}=g_{\infty}$ if $z>0$ and $\tilde{g}=0$ if $z \leqslant 0$. Then, as in Proposition 3.2, one has $\tilde{u} \leqslant \tilde{g}$ and consequently $\left.\tilde{u}\right|_{\bar{\Omega}}=u_{\infty}$ and $\tilde{u}=0$ in $\tilde{\Omega} \backslash \Omega$ implies $\partial_{z} u_{\infty}=0$ on $\Gamma_{0}$, since $\tilde{u} \in C^{1}(\tilde{\Omega})$. The free boundary is $\Phi=\partial\{\tilde{u}>0\} \cap \tilde{\Omega}=\{z=$ $\left.\phi_{\infty}(x, y)\right\}$ with $\phi_{\infty} \geqslant 0$ and analytic in $\Gamma$.

Assume, by contradiction, that $\phi_{\infty}(\bar{x}, \bar{y})=0$ for some $(\bar{x}, \bar{y}) \in \Gamma$. Then $\bar{X}=(\bar{x}, \bar{y}, 0)$ $\in \Phi \cap \Gamma_{0}$ and $u_{\infty}$ being smooth up to $\Phi$, one finds

$$
\partial_{z}^{2} u_{\infty}(\bar{X})=\Delta u_{\infty}(\bar{X})-b \partial_{z} u_{\infty}(\bar{X})=\lambda b>0 .
$$

Since $g_{\infty}^{\prime \prime}(0)=\rho_{\infty}^{\prime}(0)<\lambda b$ and $g_{\infty} \geqslant u_{\infty}$, one has $g_{\infty}>u_{\infty}>0$ in some neighborhood of $X$ in $\left\{u_{\infty}>0\right\}$, where

$$
\Delta\left(u_{\infty}-g_{\infty}\right)-b \partial_{z}\left(u_{\infty}-g_{\infty}\right) \geqslant 0
$$

holds. Whence, by Hopf's Lemma, one must have

$$
\partial_{z}\left(u_{\infty}-g_{\infty}\right)(\bar{X})<0
$$

which contradicts $\partial_{z}\left(u_{\infty}-g_{\infty}\right)(\bar{X})=\rho(0)=0$.

The next result is a quantitative result on the stability of the free boundary in terms of the given data. It is based on a technique of Brézis [B] and it extends a result of Caffarelli [C2]. 
THEOREM 3.3. Let $\phi$ and $\hat{\phi}$ be the free boundaries corresponding to the solutions $u$ and $\hat{u}$ for the data $b, g, h$ and $\hat{b}, \hat{g}, \hat{h}$, respectively, under the assumptions of Theorem 3.1. Then the following estimate holds:

$$
\int_{\Gamma}|\phi-\hat{\phi}| \leqslant \frac{1}{\lambda b}\left\{\alpha \int_{\Gamma_{1}}|g-\hat{g}|+\int_{\Gamma_{2}}|h-\hat{h}|+|b-\hat{b}| \int_{\Omega}\left(2 \lambda+\left|\partial_{z} \hat{u}\right|\right)\right\} .
$$

Proof. Since $u$ is smooth (see (3.4)) one knows that it verifies

$$
-\Delta u+b \partial_{z} u-\lambda b \chi_{\{u=0\}}=-\lambda b \quad \text { a.e. in } \Omega
$$

and $\hat{u}$ an analogous equation. Thus $w=u-\hat{u}$ verifies

$$
-\Delta w+b \partial_{z} w-\lambda b(\chi-\hat{\chi})=f-\hat{f} \quad \text { a.e. in } \Omega,
$$

where we have denoted $\chi=\chi_{\{u=0\}}, \hat{\chi}=\chi_{\{\hat{u}=0\}}$ and

$$
f-\hat{f}=(b-\hat{b})\left(\hat{\chi} \lambda-\lambda+\partial_{z} \hat{u}\right) .
$$

Now multiply equation (3.11) by the function

$$
s= \begin{cases}-1 & \text { on }\{u<\hat{u}\} \cup\{x>\hat{\chi}\} \\ 0 & \text { on }\{u=\hat{u}\} \cap\{x=\hat{\chi}\}, \\ 1 & \text { on }\{u>\hat{u}\} \cup\{x<\hat{\chi}\}\end{cases}
$$

and obtain

$$
\begin{aligned}
\lambda b \int_{\Omega}|\chi-\hat{\chi}| & =\int_{\Omega}(f-\hat{f}) s+\int_{\Omega}\left(\Delta w-b \partial_{z} w\right) s \\
& =\int_{\Omega}(f-\hat{f}) s+\int_{\Omega}\left(\Delta w-b \partial_{z} w\right) \operatorname{sign}(w) \\
& \leqslant \int_{\Omega}|f-\hat{f}|+\alpha \int_{\Gamma_{1}}|g-\hat{g}|+\int_{\Gamma_{2}}|h-\hat{h}| .
\end{aligned}
$$

The last inequality follows if we integrate by parts and approximate the $\operatorname{sign}(r)$ by smooth functions $\sigma_{\varepsilon}(r), \varepsilon>0$ satisfying $\left|\sigma_{\varepsilon}(r)\right| \leqslant 1, \sigma^{\prime}(r) \geqslant 0, \sigma_{\varepsilon}(0)=0$ and $\sigma_{\varepsilon}(r) \rightarrow$ $\operatorname{sign}(r)$ as $\varepsilon \downarrow 0$. In fact one has (with $S_{\varepsilon}(r)=\int_{0}^{r} \sigma_{\varepsilon} \geqslant 0$ )

$$
\begin{gathered}
\int_{\Omega}\left(\Delta w-b \partial_{z} w\right) \sigma_{\varepsilon}(w)=-\int_{\Omega}|\nabla w|^{2} \sigma_{\varepsilon}^{\prime}(w)-b \int_{\Omega} \partial_{z} S_{\varepsilon}(w)+\int_{\Gamma_{1} \cup \Gamma_{2}} \frac{\partial w}{\partial n} \sigma_{\varepsilon}(w) \\
\leqslant-b \int_{\Gamma_{2}} S_{\varepsilon}(w)-\alpha \int_{\Gamma_{1}} w \sigma_{\varepsilon}(w)+\alpha \int_{\Gamma_{1}}(g-\hat{g}) \sigma_{\varepsilon}(w)+\int_{\Gamma_{2}}(h-\hat{h}) \sigma_{\varepsilon}(w) \\
\leqslant \alpha \int_{\Gamma_{1}}|g-\hat{g}|+\int_{\Gamma_{2}}|h-\hat{h}| .
\end{gathered}
$$

To finish the proof we remark that

$$
\int_{\Gamma}|\phi-\hat{\phi}|=\int_{\Omega}|\chi-\hat{\chi}|
$$


4. Comparison and stability results. Next we consider the monotonic dependence of the evolutionary solution with respect to the data.

Proposition 4.1. If $u$ and $\hat{u}$ denote the solutions to the parabolic variational inequalities (2.14) and (2.15) with data $g \leqslant \hat{g}, h \leqslant \hat{h}$ and $\theta_{0} \leqslant \hat{\theta}_{0}$ then also $u \leqslant \hat{u}$ in $Q_{T}$.

Proof. As in the proof of Proposition 3.1 one gets for $w=w(t)=u(t)-\hat{u}(t)$ and a.e. $t>0$

$$
\int_{\Omega} \partial_{t} w w^{+}+\frac{1}{b} \int_{\Gamma_{2}} \partial_{t} w w^{+}+\int_{\Omega} \nabla w \cdot \nabla\left(w^{+}\right)+\int_{\Omega} \partial_{z} w w^{+}+\alpha \int_{\Omega}\left|w^{+}\right|^{2} \leqslant 0
$$

since $\theta_{0} \leqslant \hat{\theta}_{0}$ implies $f \leqslant \hat{f}$. Therefore, integrating between 0 and $t$, one finds

$$
\int_{\Omega}\left|w^{+}(t)\right|^{2}+\frac{1}{b} \int_{\Gamma_{2}}\left|w^{+}(t)\right|^{2} \leqslant 0
$$

from which the conclusion follows.

As for the elliptic problem one could also obtain frames for $u(t)$ as in Proposition 3.2, but it is perhaps more direct to compare it with steady-state solutions. As a corollary of Proposition 4.1 one gets the following result.

Proposition 4.2 . Let $(\bar{u}, \bar{\phi})$ be a steady-state solution with data $\bar{\rho}$ and $\bar{h}$ verifying the assumptions of Theorem 3.1 and (3.6)-(3.9). Suppose that the evolutionary data are such that

$$
\rho(t) \leqslant \bar{\rho}, \quad h(t) \leqslant \bar{h} \quad \text { for } t>0, \quad \text { and } \theta_{0} \leqslant \partial_{z} \bar{u} \quad \text { in } \Omega .
$$

Then the evolutionary free boundary $\Phi(t)=\partial\{u(t)>0\} \cap \Omega$ is bounded below for all $t>0$ by the free boundary $\bar{\phi}$ and it never touches the fixed boundary $\Gamma_{0}$.

Proof. From Theorem 3.2 one knows that $\bar{\phi}>0$ in $\Gamma$ and applying the transformation (2.3) to the function $\partial_{z} \bar{u}$ one obtains a function $\hat{u}(t)$ which is a solution to (2.14) and (2.15), coincides with $\bar{u}$ for all $t \geqslant l / b$ and is such that $\{\hat{u}(t)>0\}=\{\bar{u}>0\}$ for all $t>0$. Applying Proposition 4.1, one sees that $\hat{u} \geqslant u$ and this implies the conclusion.

Remark 4.1. If we assume that the evolutionary data verify the conditions

$$
\rho(t) \geqslant \underline{\rho}, \quad h(t) \geqslant \underline{h} \quad \text { for } t>0, \quad \text { and } \theta_{0} \geqslant \partial_{z} \underline{u} \quad \text { in } \Omega
$$

for some steady-state solution $\underline{u}$ corresponding to the stationary data $\rho$ and $\underline{h}$ compatible in the sense of Theorems 3.1 and 3.2, one obtains as in Proposition 4.2 that $\{u(t)>0\} \supset$ $\{\underline{u}>0\}$ and therefore one gets an upper bound for the evolutionary free boundary. Consequently if both (4.1) and (4.2) hold one can translate (3.8) for the evolutionary solution.

To extend Theorem 3.3 to the parabolic case we need some information on the evolutionary free boundary, namely that for each $t>0$ one has

$$
\Phi(t)=\left\{(x, y, z) \in \Omega: 0<z=\phi(x, y, t) \leqslant \phi_{0}(x, y)+b t\right\}=\partial\{u(t)>0\} \cap \Omega,
$$

for any $(x, y) \in \Gamma, t>0$. This will be proved in the next section under appropriate compatibility conditions on the data. 
THEOREM 4.1. Let $\phi$ and $\hat{\phi}$ be the evolutionary free boundaries for the solutions $u$ and $\hat{u}$ for the data $g, h, \theta_{0}$ and $\hat{g}, \hat{h}, \hat{\theta}_{0}$, respectively, both verifying (4.3). Then the following estimates hold:

$$
\begin{gathered}
\int_{0}^{t} \int_{\Gamma}|\phi-\hat{\phi}|<\frac{1}{\lambda b}\left\{\alpha \int_{0}^{t} \int_{\Gamma_{1}}|g-\hat{g}|+\int_{0}^{t} \int_{\Gamma_{2}}|h-\hat{h}|+l \int_{\Omega}\left|\theta_{0}-\hat{\theta}_{0}\right|+\lambda l \int_{\Gamma}\left|\phi_{0}-\hat{\phi}_{0}\right|\right\} \\
\int_{t}^{t+1} \int_{\Gamma}|\phi-\hat{\phi}| \leqslant \\
\frac{1}{\lambda b}\left\{\alpha \int_{t}^{t+1} \int_{\Gamma_{1}}|g-\hat{g}|+\int_{t}^{t+1} \int_{\Gamma_{2}}|h-\hat{h}|\right. \\
\left.+\int_{\Omega}|(u-\hat{u})(t)|+\frac{1}{b} \int_{\Gamma_{2}}|(u-\hat{u})(t)|\right\}
\end{gathered}
$$

respectively for all $t>0$ and all $t>l / b$.

Proof. Recalling (2.25) and letting $w=u-\hat{u}$ one has

$$
\partial_{t} w-\Delta w+b \partial_{z} w+q-\hat{q}=f-\hat{f} \quad \text { a.e. in } Q_{T}
$$

where $f$ and $\hat{f}$ are defined by (2.6) and

$$
q=q(t)=f(t) \chi(t)=-\lambda b \chi(t) \text { and } \hat{q}=-\lambda b \hat{\chi}
$$

with $\chi(t)$ and $\hat{\chi}(t)$ being the characteristic functions of the sets $\{u(t)=0\}$ and $\{\hat{u}(t)=0\}$ respectively (recall that (4.3) implies $\left\{z>\phi_{0}(x, y)+b t\right\} \subset\{u>0\}$ and therefore $\chi \chi_{\mathcal{O}}=0$; analogously $\hat{\chi} \hat{\chi}_{\mathcal{O}}=0$ ).

Multiplying equation (4.6) by the function $s$ given in (3.12) and integrating on $\Omega \times] 0, t$, one concludes (as in the proof of Theorem 3.3)

$$
\begin{aligned}
& \lambda b \int_{0}^{t} \int_{\Omega}|\chi-\hat{\chi}|=\int_{0}^{t} \int_{\Omega}(f-\hat{f}) s+\int_{0}^{t} \int_{\Omega}\left(\Delta w-b \partial_{z} w-\partial_{t} w\right) s \\
& \quad \leqslant b \int_{0}^{l / h} \int_{\Omega}\left|\theta \chi_{\mathcal{O}}-\hat{\theta} \hat{\chi}_{\mathcal{O}}\right|+\lambda b \int_{0}^{l / h} \int_{\Omega}\left|\chi_{\mathcal{O}}-\hat{\chi}_{\mathcal{O}}\right|+\alpha \int_{0}^{t} \int_{\Gamma_{1}}|g-\hat{g}|+\int_{0}^{t} \int_{\Gamma_{2}}|h-\hat{h}|,
\end{aligned}
$$

since we have for the new terms, for any $\tau>\sigma \geqslant 0$,

$$
\begin{aligned}
\int_{\sigma}^{\tau} \int_{\Omega} \partial_{t} w s+\frac{1}{b} \int_{\sigma}^{\tau} \int_{\Gamma_{2}} \partial_{t} w s & =\int_{\sigma}^{\tau} \int_{\Omega} \partial_{t} w \operatorname{sign}(w)+\frac{1}{b} \int_{\sigma}^{\tau} \int_{\Gamma_{2}} \partial_{t} w \operatorname{sign}(w) \\
& =\int_{\Omega}(|w(\tau)|-|w(\sigma)|)+\frac{1}{b} \int_{\Gamma_{2}}(|w(\tau)|-|w(\sigma)|) \\
& \geqslant-\int_{\Omega}|w(\sigma)|-\frac{1}{b} \int_{\Gamma_{2}}|w(\sigma)|
\end{aligned}
$$

and $w(0)=0$. To show (4.5), integrate in $\Omega \times] t, t+1$ [ and recalling that $f=\hat{f}=-\lambda b$ for all $t>l / b$, use (4.7) with $\sigma=t$ and $\tau=t+1$, and conclude as before.

As for the steady-state case we need to know in what sense the inverse transformation (2.4) can be performed in order to obtain the solution of the initial Problem (C). A first step is to show that the temperature $\theta=\partial_{t} u / b+\partial_{z} u$ is a nonnegative function. This fact has important consequences in the further analysis of the free boundary, namely that $\mathcal{O} \subset\{u>0\}$. 
THEOREM 4.2. Under the assumptions of Theorem 2.1, one has

$$
\partial_{t} u+b \partial_{z} u \geqslant 0 \quad \text { a.e. in } Q_{T} .
$$

Proof. We need the following family of approximating solutions $u_{\varepsilon}$ to the solution $u$ of (2.14) and (2.15):

$$
\begin{aligned}
& \partial_{t} u_{\varepsilon}-\Delta u_{\varepsilon}+b \partial_{z} u_{\varepsilon}+\beta_{\varepsilon}\left(u_{\varepsilon}\right)=f \text { in } Q_{T}, \quad u_{\varepsilon}(0)=0 \quad \text { in } \Omega ; \\
& u_{\varepsilon \mid \Gamma_{0}}=0, \quad-\partial u_{\varepsilon} /\left.\partial n\right|_{\Gamma_{1}}=\alpha\left(u_{\varepsilon}-g\right), \quad \partial_{t} u_{\varepsilon} / b+\left.\partial_{z} u_{\varepsilon}\right|_{\Gamma_{2}}=0, \quad \text { for } t>0
\end{aligned}
$$

where $\varepsilon>0$ and $\beta_{\varepsilon}$ is a one-parameter family of smooth functions such that

$$
\beta_{\varepsilon}(r)=0 \text { for } r \geqslant \varepsilon, \quad \beta_{\varepsilon}(0)=-\lambda b \quad \text { and } \quad \beta_{\varepsilon}^{\prime}(r)>0 \text { for } r<\varepsilon .
$$

By classical monotonicity techniques (see [L1]) one can obtain the existence of a unique solution $u_{\varepsilon}$ in the same spaces as for $u$ (see (2.24)) and that $u_{\varepsilon} \rightarrow u$ as $\varepsilon \rightarrow 0$. An important step is to show $u_{\varepsilon} \geqslant 0$ in $Q_{T}$ (therefore $-\lambda b \leqslant \beta_{\varepsilon}\left(u_{\varepsilon}\right) \leqslant 0$ ). In fact, multiplying (4.9) by $-u_{\varepsilon}^{-}=\min \left(u_{\varepsilon}, 0\right)$ and integrating over $\left.\Omega \times\right] 0, t\left[\right.$ one concludes $u_{\varepsilon}^{-}=0$ from

$$
\begin{array}{r}
\frac{1}{2} \int_{\Omega}\left|u_{\varepsilon}^{-}(t)\right|^{2}+\frac{1}{2 b} \int_{\Gamma_{2}}\left|u_{\varepsilon}^{-}(t)\right|^{2}+\int_{0}^{t} \int_{\Omega}\left|\nabla u_{\varepsilon}^{-}\right|^{2}+\frac{b}{2} \int_{0}^{t} \int_{\Gamma_{2}}\left|u_{\varepsilon}^{-}\right|^{2}+\alpha \int_{0}^{t} \int_{\Gamma_{1}}\left|u_{\varepsilon}^{-}\right|^{2} \\
=\iint_{\left\{u_{\varepsilon}<0\right\}}\left(f-\beta_{\varepsilon}\left(u_{\varepsilon}\right)\right)\left(-u_{\varepsilon}^{-}\right)-\alpha \int_{0}^{t} \int_{\Gamma_{1}} g u_{\varepsilon}^{-}-\int_{0}^{t} \int_{\Gamma_{2}} h u_{\varepsilon}^{-} \leqslant 0,
\end{array}
$$

since $g, h \geqslant 0$ and $-\beta_{\varepsilon}\left(u_{\varepsilon}\right)>\lambda b$ in the region where $u_{\varepsilon}<0$.

Now it is enough to show that $w=\partial_{t} u_{\varepsilon}+b \partial_{z} u_{\varepsilon} \geqslant 0$ for any $\varepsilon>0$, which we shall do by remarking that $w$ satisfies the following linear equation with $d_{\varepsilon}(X, t)=\beta_{\varepsilon}^{\prime}\left(u_{\varepsilon}(X, t)\right) \geqslant$ 0 ,

$$
\partial_{t} w-\Delta w+b \partial_{z} w+d_{\varepsilon} w=0 \text { in } Q_{T},
$$

and the following conditions for a.a. $t>0$,

$$
\begin{gathered}
w=b \partial_{z} u_{\varepsilon} \geqslant 0 \quad \text { on } \Gamma_{0}, \quad w=b h \geqslant 0 \text { on } \Gamma_{2}, \\
-\partial w / \partial n=\alpha(w-b \rho) \quad \text { on } \Gamma_{1}
\end{gathered}
$$

and from (4.9) the initial condition

$$
w(0)=\partial_{t} u_{\varepsilon}(0)=f(0)+\lambda b \geqslant 0 \quad \text { in } \Omega .
$$

Then multiplying (4.12) by $-w^{-}$and taking the conditions (4.13)-(4.15) into account, one obtains $w \geqslant 0$ from

$$
\begin{aligned}
\frac{1}{2} \int_{\Omega}\left|w^{-}(t)\right|+\int_{0}^{t} \int_{\Omega}\left|\nabla w^{-}\right|^{2}+\int_{0}^{t} \int_{\Omega} d_{\varepsilon}\left|w^{-}\right|^{2} & +\alpha \int_{0}^{t} \int_{\Gamma_{1}}\left|w^{-}\right|^{2} \\
& =\alpha \int_{0}^{t} \int_{\Gamma_{1}} \rho(-w)^{-} \leqslant 0 .
\end{aligned}
$$

5. Analysis of the evolutionary free boundary. The analysis of the regularity of the free boundary associated with a parabolic unilateral variational inequality was done first by Caffarelli $[\mathbf{C 1}]$ for the one-phase Stefan problem (see [F], particularly Ch. 2, §9, and also 
the earlier special case of [FK] with a star-shaped geometry). More recently Kröner [K] has extended that analysis to the free boundary of the nonsteady flow of a compressible fluid through a porous dam, where the existence of a monotonicity property in one direction allows important simplifications in the proofs. Since our problem presents an analogous feature, we discuss in this section how their arguments can be applied to the continuous casting Stefan problem.

First we assume that the free boundary to (2.14), (2.15) exists and does not touch the fixed boundaries $\Gamma_{0}$ and $\Gamma_{2}$ :

$$
\partial\{u(t)>0\} \cap \Gamma_{0}=\varnothing \quad \text { and } \quad \partial\{u(t)=0\} \cap \Gamma_{2}=\varnothing \quad \text { for all } t>0 .
$$

We have seen in the preceding section how it is possible to assure (5.1) by means of comparison arguments and reasonable assumptions on the data. We also remark, as in the steady-state problem, that if $u(t)>0$ in $\Omega$ the equivalence between $u$ and $\theta$ loses its sense, since then conditions (1.2) and (1.3) are excessive.

Together with the inequality (4.8), another crucial property that we shall consider is

$$
\partial_{z} u \geqslant 0 \text { in } \Omega, \text { for all } t>0 .
$$

This condition is more delicate to show than in the steady case of Theorem 3.1 and we shall give in Proposition 5.1 below sufficient assumptions to obtain it. That allows us to define the function $\phi$ by

$$
\phi(x, y, t)=\inf \{z: u(x, y, z, t)>0\} \quad \text { for all }(x, y) \in \Gamma, t>0,
$$

which, by the continuity of $u$, is an upper semicontinuous function in $\Gamma$, for each fixed $t$.

THEOREM 5.1. Under the conditions of Theorem 2.1, assume the initial free boundary $\phi_{0} \in C^{1}(\Gamma)$ and that (5.1) and (5.2) hold. Then the free boundary is represented as a graph (seee (4.3) and (5.3)) of a function $\phi \in C^{1}\left(\Gamma_{T}\right)$, for each $T<\infty$, with $\partial_{t} \phi \leqslant b$. Moreover, $(\theta, \phi)$ is the classical solution to Problem (C).

Proof. Since the proof is essentially the same as that of Theorem 1.5 of $[\mathbf{K}]$ we refer only to the main steps and the necessary adaptations to our situation (see Remark 7.1).

First, observe that $\partial_{t} u+b \partial_{z} u \geqslant 0$ implies $\partial_{t} \phi \leqslant b$. In fact, introducing the transformation

$S: \Gamma \times] 0, l[\times] 0, T[\ni(x, y, z, t) \mapsto(x, y, z-b t, t) \in \Gamma \times]-b T, l-b T[\times] 0, T[$ and defining accordingly

$$
v(x, y, z, t)=u(x, y, z+b t, t) \text { and } \psi(x, y, t)=\phi(x, y, t)-b t \text {, }
$$

one finds $\partial_{t} v \geqslant 0$ and $\{X: v(x, t)>0\}=\{X: z>\psi(x, y, t)\}$; hence $\psi(x, y, t) \leqslant$ $\psi(x, y, \tau)$ for $t>\tau$ and $\partial_{t} \phi-b=\partial_{t} \psi \leqslant 0$.

Now consider for any $\Gamma^{\prime} \subset \subset \Gamma, 0<\sigma<\tau \leqslant T$, the subsets

$$
\begin{aligned}
G & =\left\{(x, y, z, t): \sigma<t<\tau,(x, y) \in \Gamma^{\prime}, \psi(x, y, t)<z<l-b t\right\}, \\
\Psi & =\{(x, y, z, t) \in \bar{G}: z=\psi(x, y, t)\}
\end{aligned}
$$

and, using the regularity of $u$, one has $v, \nabla v \in C^{0}(\bar{G}), v$ may be extended by zero in a neighborhood $\tilde{G}$ of $G \cup \Psi$ such that $v \in W_{p}^{2,1}(\tilde{G}), \forall p<\infty$, and it verifies

$$
\begin{aligned}
& \partial_{t} v-\Delta v=-\lambda b<0 \text { in } G, \\
& v=|\nabla v|=0 \text { in } \Psi, \\
& v>0, \partial_{z} v>0 \text { and } \partial_{t} v>0 \quad \text { in } \bar{G} \backslash \Psi .
\end{aligned}
$$


Moreover, it is possible (see Theorem 2.5 of $[\mathbf{K}]$ or Proposition 6.1 below) to prove the local boundedness of $\partial_{t} u$; so also

$$
\sup _{G}\left|\partial_{t} v\right| \leqslant M
$$

and we can apply Theorem 3.1 of [K] (see also Remark 7.1 below) in order to conclude the local Lipschitz continuity of the function $\psi$ both in space and time variables.

Consequently $\phi \in C_{\mathrm{loc}}^{0.1}\left(\Gamma_{T}\right)$ and the further regularity of $\phi$, as well as the local continuity of the temperature $\theta$ and its derivatives up to free boundary, follow by the arguments of Caffarelli (see [C1], [F] or [K], §4). Finally, by Proposition 2.1, one concludes the existence (and uniqueness) of the solution for Problem (C).

Remark 5.1. The assumption $\phi_{0} \in C^{1}(\Gamma)$ and the argument of Lemma 2.2 of [K] allow us to conclude that in fact

$$
\phi(x, y, t)<\phi_{0}(x, y)+b t, \quad \text { for all } t>0,(x, y) \in \Gamma .
$$

Moreover, since at $t=0$ one has $\theta(0)=\partial_{t} u(0)=\partial_{t} v(0)$, the argument of Lemma 9.3 of $[\mathbf{F}]$ yields the continuity of the temperature at the initial instant.

To discuss the condition (5.2), we recall that the boundary condition (2.12) for $u$ on $\Gamma_{2}$ requires $\partial_{t} u \leqslant b h$ there. So, in general, we can not choose the boundary temperatures arbitrarily but they must satisfy some appropriate conditions.

Proposition 5.1. Let, in addition to the assumptions of Theorem 2.1 and (2.28), the following compatibility conditions hold:

$$
\begin{gathered}
\partial_{t} h-\Delta_{x y} h \geqslant 0 \quad \text { in } \Gamma_{T}, \quad h(x, y, 0) \geqslant \sup _{0<z<1} \theta_{0}(x, y, z) \text { in } \Gamma, \\
\partial h / \partial n+\alpha h \geqslant \alpha \rho(l, t), \quad \rho(0, t)=0 \quad \text { on } \partial \Gamma \text { and } \partial_{z} \rho \geqslant 0 \quad \text { for } t>0 .
\end{gathered}
$$

Then one has

$$
\partial_{t} u \leqslant b h \text { and } \partial_{z} u \geqslant 0 \text { in } Q_{T} .
$$

Proof. As in the proof of Theorem 4.2 we shall use a family of approximating solutions $\tilde{u}_{\varepsilon}$ for the variational inequality (2.14), (2.15) in the form (4.9), (4.10), but now with $\beta_{\varepsilon}$ replaced by $\tilde{\beta}^{\varepsilon}$, with $\tilde{\beta}_{\varepsilon}(r)=\beta_{\varepsilon}(r+\varepsilon)$, so that $\tilde{\beta}_{\varepsilon}(0)=0$, with $\tilde{u}_{\varepsilon \mid \Gamma_{0}}=-\varepsilon$ and $f$ replaced by some regularization $f_{\varepsilon} \rightarrow f$ as $\varepsilon \downarrow 0$ with $\partial_{t} f_{\varepsilon} \leqslant 0$ and $f_{\varepsilon}(0) \leqslant f(0)$ (see Lemma 2.2). Since $\tilde{u}_{\varepsilon} \rightarrow u$ also, it is enough to prove that $\partial_{t} \tilde{u}_{\varepsilon} \leqslant b h$ in $Q_{T}$. Now $w=\partial_{t} \tilde{u}_{\varepsilon}$ is the solution of the linear mixed problem

$$
\begin{gathered}
\partial_{t} w-\Delta w+b \partial_{z} w+\tilde{d}_{\varepsilon} w=\partial_{t} f_{\varepsilon} \quad \text { in } Q_{T}, \quad w(0)=f_{\varepsilon}(0) \quad \text { in } \Omega \\
w\left|\Gamma_{0}=0, \quad-\partial w / \partial n\right|_{\Gamma_{1}}=\alpha\left(w-\partial_{t} g\right), \quad \partial_{t} w+\left.b \partial_{z} w\right|_{\Gamma_{2}}=b \partial_{t} h, t>0,
\end{gathered}
$$

with $\tilde{d}_{\varepsilon}(x, t)=\tilde{\beta}_{\varepsilon}^{\prime}\left(\tilde{u}_{\varepsilon}(X, t)\right)=\beta_{\varepsilon}^{\prime}\left(\tilde{u}_{\varepsilon}(X, t)+\varepsilon\right) \geqslant 0$. Again by the weak maximum principle, it is easy to see that a function $v$ satisfying the conditions

$$
\begin{gathered}
v \geqslant 0 \quad \text { in } Q_{T}, \quad v(0) \geqslant f(0) \text { in } \Omega, \\
\partial_{t} v+\Delta v+b \partial_{z} v \geqslant 0 \quad \text { in } Q_{T}, \\
\partial v / \partial n+\left.\alpha v\right|_{\Gamma_{1}} \geqslant \alpha \partial_{t} g \text { and } \partial_{t} v+\left.b \partial_{z} v\right|_{\Gamma_{2}} \geqslant b \partial_{t} h \quad \text { for } t>0
\end{gathered}
$$

is a supersolution to (5.7), (5.8), so that it verifies $v \geqslant w$ in $Q_{T}$. 
Clearly $v=b h$ satisfies (5.9), since (5.5) implies, in particular,

$$
\begin{aligned}
\partial_{t} \int_{0}^{t} \rho(x, y, z+b(\tau-t), \tau) d \tau & =\rho(x, y, z, t)-b \int_{0}^{t} \partial_{z} \rho(x, y, z+b(\tau-t), \tau) d \tau \\
& \leqslant \rho(x, y, l, t) .
\end{aligned}
$$

Next, we use a similar argument to prove that $\partial_{z} u \geqslant 0$. As in the proof of Theorem 4.2 (now arguing with $\left(\tilde{u}_{\varepsilon}+\varepsilon\right)^{-}$) one can show that $\tilde{u}_{\varepsilon} \geqslant-\varepsilon$ in $Q_{T}$ and therefore $\partial_{z} \tilde{u}_{\varepsilon} \geqslant 0$ on $\Gamma_{0}$ (since $\tilde{u}_{\varepsilon \mid \Gamma_{0}}=-\varepsilon$ ). Hence $\zeta=\partial_{z} \tilde{u}_{\varepsilon}$ satisfies

$$
\begin{gathered}
\partial_{t} \zeta-\Delta \zeta+b \partial_{z} \zeta+\tilde{d}_{\varepsilon} \zeta=\partial_{z} f_{\varepsilon} \geqslant 0 \quad \text { in } Q_{T}, \quad \zeta(0)=0 \quad \text { in } \Omega, \\
\left.\zeta\right|_{\Gamma_{0} \cup \Gamma_{2} \geqslant 0} \text { and } \partial \zeta / \partial n+\left.\alpha \zeta\right|_{\Gamma_{1}} \geqslant 0 \quad \text { for } t>0
\end{gathered}
$$

by recalling (2.29) and (5.5), which last part implies $\partial_{z} g \geqslant 0$.

Once more by the weak maximum principle one finds $\zeta \geqslant 0$, concluding the proof of (5.6).

6. Asymptotic behavior as $\mathbf{t} \rightarrow+\infty$. Another advantage of the variational formulation to the continuous casting problem is the possibility of the direct application of the general results to the asymptotic behavior of strong solutions to monotone parabolic variational inequalities (see [R3]). This fact, which has already been observed by Brière $[\mathbf{B r}]$ in a particular case, immediately yields various stabilization results of the variational solution of (2.14), (2.15) with respect to the stationary one of (3.2), depending on the corresponding assumptions on the data, namely on the quantities

$$
\begin{aligned}
& \xi(t)=\int_{t}^{t+1} \int_{\Gamma_{1}}\left|g(\tau)-g_{\infty}\right|^{2}+\int_{t}^{t+1} \int_{\Gamma_{2}}\left|h(\tau)-h_{\infty}\right|^{2}, \\
& \eta(t)=\int_{t}^{t+1} \int_{\Gamma_{1}}\left|\partial_{t} g(\tau)\right|^{2}+\int_{t}^{t+1} \int_{\Gamma_{2}}\left|\partial_{t} h(\tau)\right|^{2} .
\end{aligned}
$$

Furthermore, one has the stability results for the free boundary, particularly (4.5) for $u=\hat{u}_{\infty}$ and $\phi=\hat{\phi}_{\infty}$ for $t>l / b$, or under the stronger convergence when $\partial_{t} u(t) \rightarrow 0$ in $L^{2}(\Omega) \times L^{2}\left(\Gamma_{2}\right)$ as $t \rightarrow \infty$ the estimate (3.10) with $\phi=\phi_{\infty}, \hat{\phi}=\phi(t), g=g_{\infty}, \hat{g}=g(t)$, $h=h_{\infty}, \hat{h}=h(t)-\partial_{t} u(t) / b, b=\hat{b}$ and the additional term $\int_{\Omega}\left|\partial_{t} u(t)\right|$ on the right side, that is, considering the parabolic problem for large $t$ as a perturbation of the elliptic one. Hence if we assume $\phi_{\infty}>0$ and $\phi(t)>0$ for all large $t$ one obtains not only the asymptotic stabilization of the free boundary but also its order of convergence. Therefore, taking into account Theorems 1, 2, and 3 of [R3] and Theorems 4.1 and 3.3, one can state the following results.

TheOREM 6.1. Let $(u(t), \phi(t))$ and $\left(u_{\infty}, \phi_{\infty}\right)$ be the solutions and the free boundaries for the evolutionary and stationary problems, respectively. One has the following asymptotic results for $t \rightarrow \infty$ :

(i) If $\xi(t) \rightarrow 0$ (resp. $\xi(t)=O\left(t^{-\alpha}\right)$ or $\left.\xi(t)=O\left(e^{-\alpha t}\right)\right)$ then

$$
\begin{gathered}
u(t) \rightarrow u_{\infty} \text { in } H \simeq L^{2}(\Omega) \times L^{2}\left(\Gamma_{2}\right) \quad \text { and } \quad \int_{t}^{t+1} \int_{\Gamma}\left|\phi(\tau)-\phi_{\infty}\right| \rightarrow 0 ; \\
\left\|u(t)-u_{\infty}\right\|_{H}+\int_{t}^{t+1} \int_{\Gamma}\left|\phi(\tau)-\phi_{\infty}\right|=O\left(t^{-\alpha / 2}\right) \quad\left(\text { resp. } O\left(e^{-\beta t}\right)\right)
\end{gathered}
$$


for any $\alpha>0$ and for some $\beta>0, \beta<\alpha$.

(ii) If $\mu(t) \equiv \xi(t)+\eta(t) \rightarrow 0$ (resp. $\mu(t)=O\left(t^{-\alpha}\right)$ or $\mu(t)=O\left(e^{-\alpha t}\right)$ ) then

$$
\begin{gathered}
u(t) \rightarrow u_{\infty} \text { in } V \cap i i_{\mathrm{loc}}^{2}(\Omega), \quad \partial_{t} u(t) \rightarrow 0 \text { in } H \quad \text { and } \int_{\Gamma}\left|\phi(t)-\phi_{\infty}\right| \rightarrow 0 ; \\
\left\|u(t)-u_{\infty}\right\|_{V \cap H_{\mathrm{loc}}^{2}}+\left\|\partial_{t} u(t)\right\|_{H}+\int_{\Gamma}\left|\phi(t)-\phi_{\infty}\right|=O\left(t^{-\alpha / 2}\right) \quad\left(\text { resp. } O\left(e^{-\gamma t}\right)\right)
\end{gathered}
$$

for any $\alpha>0$ and for some $\gamma>0, \gamma<\alpha$. Of course, the results on $\phi$ require $\phi_{\infty}>0$ and $\phi(t)>0$ for $t$ large.

Remark 6.1. In the case (ii) we also have the asymptotic convergence of the temperature as $t \rightarrow \infty$ :

$$
\theta(t)=\partial_{t} u(t) / b+\partial_{z} u(t) \rightarrow \partial_{z} u_{\infty}=\theta_{\infty} \quad \text { in } L^{2}(\Omega),
$$

and, naturally, also the corresponding rates for the $L^{2}$-convergence.

In the proof of Theorem 5.1 we referred to the need for local boundedness of $\partial_{t} u$ which can be proved by local estimates as in $[\mathbf{K}]$. However, in our case, with some mild additional assumptions on the data, namely

$$
\partial_{t} h \in L^{\infty}\left(\Gamma_{2} \times\right] 0, \infty[) \text { and } \rho, \partial_{z} \rho \in L^{\infty}\left(\Gamma_{1} \times\right] 0, \infty[),
$$

one can give a global $L^{\infty}$ estimate for $\partial_{t} u$, which can be used to improve the asymptotic convergence of the temperature and the free boundary, and has an intrinsic interest in view of the boundary conditions (5.8).

Proposition 6.1. Under the assumptions of Theorem 2.1, if in addition (6.6) holds, one has

$$
\partial_{t} u \in L^{\infty}(\Omega \times] 0, \infty[) .
$$

Proof. We consider again the linear mixed problem (5.7), (5.8) where we have chosen $\partial_{t} f_{\varepsilon}$ given for any $v \in V$ by (see Lemma 2.2)

$$
\left\langle\partial_{t} f_{\varepsilon}(t), v\right\rangle=-b^{2} \int_{\Omega} v q_{\varepsilon}(t) \partial_{z} \theta_{0}(z-b t)+\lambda b^{2} \int_{\Omega} q_{\varepsilon}(t) \partial_{z} v-\lambda b^{2} \int_{\Gamma_{2}} q_{\varepsilon}(t) v \quad(t>0),
$$

$0 \leqslant q_{\epsilon}(t) \leqslant \chi_{\mathcal{O}}(t) \leqslant 1$ being a family of regularizations of the characteristic function of the set $\mathcal{O}$ defined in (2.5). Since $\partial_{t} f_{\varepsilon}(t)=0$ for any $t \geqslant l / b$, we estimate first the $L^{\infty}$-norm of $w=\partial_{t} \tilde{u}_{\varepsilon}$ in the finite interval $\left[0, T_{*}\right], T_{*}=l / b$ and then the $L^{\infty}$ estimate in the remainder $\left[T_{*}, \infty\left[\right.\right.$ will follow easily. Using the fact $\tilde{d}_{\varepsilon} \geqslant 0$ in (5.7), the estimates we get are independent of $\varepsilon$.

The method of proof is classical and is essentially discussed in [LSU], Ch. III, §7, and so we only sketch it, making the necessary changes in order to take into account the mixed boundary conditions (5.8) (compare with the elliptic version of this estimate in [MS]).

Notice that assumptions (2.21) and (6.6) imply the existence of a constant $C_{0}>0$, independent of $\varepsilon$ and $t$, such that

$$
\left\|f_{\varepsilon}(0)\right\|_{L^{\infty}(\Omega)}+\left\|\partial_{t} g(t)\right\|_{L^{\infty}\left(\Gamma_{1}\right)}+\left\|\partial_{t} h(t)\right\|_{L^{\infty}\left(\Gamma_{2}\right)} \leqslant C_{0} .
$$


Now we consider $w^{k}=(w-k)^{+}$and $w_{k}=-(w+k)^{-}$for $k \geqslant C_{0}$; we multiply them by the equation of (5.7) and we integrate in $Q_{T}$. Taking (6.8) and (5.8) into account, we have for any $t>0$

$$
\begin{aligned}
& \int_{0}^{t} \int_{\Omega} \partial_{t} w w^{k}+\frac{1}{b} \int_{0}^{t} \int_{\Gamma_{2}} \partial_{t} w w^{k}+\int_{0}^{t} \int_{\Omega}\left\{\nabla w \cdot \nabla w^{k}+b \partial_{z} w w^{k}+\tilde{d}_{\varepsilon} w w^{k}\right\}+\alpha \int_{0}^{t} \int_{\Gamma_{1}}(w-k) w^{k} \\
&=b^{2} \int_{0}^{t} \int_{\Omega}\left\{\lambda q_{\varepsilon} \partial_{z} w^{k}-q_{\varepsilon} \partial_{z} \theta_{0} w^{k}\right\}+\alpha \int_{0}^{t} \int_{\Gamma_{1}}\left(\partial_{t} g-k\right) w^{k}+\int_{0}^{t} \int_{\Gamma_{2}}\left(\partial_{t} h-\lambda b^{2} q_{\varepsilon}\right) w^{k} .
\end{aligned}
$$

Denoting $A_{k}(t)=\{X \in \bar{\Omega}: w(t)>k\}$ and observing that

$$
\frac{1}{b} \int_{0}^{t} \int_{\Gamma_{2}} \partial_{t} w w^{k}+\int_{0}^{t} \int_{\Omega} \tilde{d}_{\varepsilon} w w^{k}=\frac{1}{2 b} \int_{\Gamma_{2}}\left(w^{k}\right)^{2}(t)+\int_{0}^{t} \int_{A_{k}(t)} \tilde{d}_{\varepsilon} w(w-k) \geqslant 0
$$

one obtains (recalling $\left|q_{\varepsilon}\right| \leqslant 1$ and the Schwartz inequality)

$$
\begin{aligned}
\int_{\Omega}\left(w^{k}\right)^{2}(t)+\int_{0}^{t} \int_{\Omega}\left|\nabla w^{k}\right|^{2}+\frac{b}{2} \int_{0}^{t} \int_{\Gamma_{2}}\left(w^{k}\right)^{2}+\alpha \int_{0}^{t} \int_{\Gamma_{1}}\left(w^{k}\right)^{2} & \\
\leqslant \int_{0}^{t} \int_{A_{k}(t)} b^{4}\left(\lambda^{2}+C_{1}^{2} C_{0}^{2}\right)+\frac{1}{\alpha} \int_{0}^{t} \int_{\Gamma_{1} \cap A_{k}(t)}\left(k+C_{0}\right)^{2} & +\frac{2}{b} \int_{0}^{t} \int_{\Gamma_{2} \cap A_{k}(t)}\left(C_{0}+\lambda b^{2}\right)^{2}
\end{aligned}
$$

where $C_{1}$ is Poincaré's constant (i.e., $\|v\|_{L^{2}(\Omega)} \leqslant C_{1}\|\nabla v\|_{L^{2}(\Omega)}, \forall v \in V$ ).

Now we can finish the argument as in [LSU], pp. 184-185, since from (6.9) and Hölder's inequality one obtains an estimate of the type

$$
\left|w^{k}\right|^{2} Q_{T_{*}} \equiv \sup _{0<t<T_{*}} \int_{\Omega}\left(w^{k}\right)^{2}(t)+\int_{0}^{T_{*}} \int_{\Omega}\left|\nabla w^{k}\right|^{2} \leqslant C k^{2} \mu^{2(1+\gamma) / r}(k)
$$

for $k=\max \left(1, C_{0}\right)$ and where

$$
\mu(k)=\int_{0}^{T_{*}}\left\{\operatorname{mes}_{\Omega}^{r / q} A_{k}(t)+\operatorname{mes}_{\partial \Omega}^{r / q}\left[A_{k}(t) \cap \Gamma_{1}\right]+\operatorname{mes}_{\partial \Omega}^{r / q}\left[A_{k}(t) \cap \Gamma_{2}\right]\right\} d t
$$

for some appropriate numbers $r, q \geqslant 2$ and $\gamma>1$. Then using the inequalities of $\mathrm{Ch}$. II, $\S 6$ of [LSU] one can deduce that $w \leqslant M$ for a certain constant $M>C_{0}$ depending on fixed quantities. Analogously one concludes $w \geqslant-M$.

Finally, considering the function $v=v(z)=M z+M$, from (5.9) one immediately finds that $v$ is a supersolution to (5.7), (5.8) in $\Omega \times] T_{*}, \infty[$. Since $-v$ is also a supersolution in that set, one concludes that $w=\partial_{t} \tilde{u}_{\varepsilon}$ is bounded in $Q_{\infty}$ independently of $\varepsilon$ and therefore (6.7) is proved.

Corollary 6.1. If in addition to (6.6) one has $\mu(t) \rightarrow 0$ in Theorem 6.1, one has for $t \rightarrow \infty$

$$
u(t) \rightarrow u_{\infty} \text { in } W_{\mathrm{loc}}^{2, p}(\Omega) \cap C^{1, \alpha}(\Omega), \quad \forall p<\infty, \forall 0 \leqslant \alpha<1,
$$

and the temperature $\theta(t) \rightarrow \theta_{\infty}$ in $L \mathrm{Poc}(\Omega)$ (see (6.5)). 
Proof. Since $\partial_{t} u(t) \rightarrow 0$ in $L^{2}(\Omega)$, from estimate (6.7) one has the uniform integrability in $\Omega$ of $\left|\partial_{t} u(t)\right|^{p}$ for any $p<\infty$ and $t>0$ and hence also $\partial_{t} u(t) \rightarrow 0$ in $L^{p}(\Omega)$. Now (6.10) follows from (6.4), the linear $L^{p}$-elliptic estimates and the Rellich-Kondratchoff theorem.

7. The strong stabilization of the free boundary. The results of Theorem 6.1(ii) give a stabilization of the free boundary in $L^{1}(\Gamma)$-norm. In view of the regularity results of Theorems 3.1 and 5.1, one easily conjectures a stronger convergence. Actually, we show in this section how an interpolation argument can give a convergence in $C^{0, \alpha}(\Gamma)$, provided one assures a local $L^{\infty}$ estimate for the spatial gradient of $\phi(t)$, uniformly in $t$. This technique has been used already in $[\mathbf{R 2}]$ to discuss the stability of the free boundary in the incompressible evolutionary dam problem, where that estimate was simpler to obtain. In the next proposition we shall adapt the technique used by Kröner $[\mathbf{K}]$ and already evoked in the proof of Theorem 5.1. (See also [A], [KS], p. 255, or [F], p. 177.)

Proposition 7.1. Under the conditions of Theorem 5.1 and Corollary 6.1 one has

$$
\sup _{t>1 / h} \sup _{(x, y) \in \Gamma^{\prime}}\left\{\left|\partial_{x} \phi(x, y, t)\right|+\left|\partial_{y} \phi(x, y, t)\right|\right\} \leqslant C
$$

for some constant $C=C\left(\Gamma^{\prime}\right)>0$ and any open subset $\Gamma^{\prime} \subset \subset \Gamma$.

Proof. For any $\Gamma^{\prime} \subset \subset \Gamma$, let $\tilde{\Gamma}^{\prime}$ be an open set such that $\Gamma^{\prime} \subset \tilde{\Gamma}^{\prime} \subset \Gamma$ with $d\left(\tilde{\Gamma}^{\prime}, \partial \Gamma\right)$ $>0$ and $d\left(\Gamma^{\prime}, \partial \tilde{\Gamma}^{\prime}\right)>0$, and define for $\delta>\tilde{\delta}>0$, arbitrarily small,

$$
\left.\tilde{\Omega}^{\prime}=\tilde{\Gamma}^{\prime} \times\right] \tilde{\delta}, l-\tilde{\delta}\left[\text { and } \Omega^{\prime}=\Gamma^{\prime} \times\right] \delta, l-\delta[.
$$

Taking into account the assumptions on the free boundaries, it is not restrictive to assume that for $\delta$ small enough and for all $t>0$

$$
\left\{X \in \Omega: z=\phi(x, y, t),(x, y) \in \Gamma^{\prime}\right\} \subset \Omega^{\prime} \subset \tilde{\Omega}^{\prime} .
$$

Denote

$$
\begin{aligned}
& G=\left\{(X, t) \in \tilde{\Omega}^{\prime} \times\right] l / b, t[: z>\phi(x, y, t)\}, \\
& \Phi=\left\{(X, t) \in \tilde{\Omega}^{\prime} \times\right] l / b, \infty[: z=\phi(x, y, t)\}, \\
& L=\partial G \backslash \Phi, \\
& F=\left\{(X, t) \in \Omega^{\prime} \times\right] l / b+\delta, \infty[: z \geqslant \phi(x, y, t)\},
\end{aligned}
$$

and remark that $F \subset G \cup \Phi$ and $d(F, L)>0$.

Now we claim that there exist fixed constants $A_{0}, \varepsilon_{0}>0$ independent of $t$, such that

$$
w \equiv u+\varepsilon \partial_{x} u+\varepsilon \partial_{y} u-A \partial_{z} u \leqslant 0 \quad \text { in } F, \quad \forall A \geqslant A_{0},|\varepsilon| \leqslant \varepsilon_{0} .
$$

Fix a constant $m>0$ such that

$$
-m\left(\partial_{t} \zeta-\Delta \zeta+b \partial_{z} \zeta\right) \leqslant \lambda b \quad \text { in } G
$$

for some function $\zeta \in C^{2}\left(\mathbf{R}^{3} \times \mathbf{R}_{+}\right)$verifying $\zeta \geqslant 0, \zeta=0$ in $F$ and $\zeta \geqslant 1$ on $L$ ( $m$ depends only on $\Omega^{\prime}, \tilde{\Omega}^{\prime}$, and $\delta$ ).

Recalling (2.25) and $f=-\lambda b$ for $t \geqslant l / b$ one has

$$
\partial_{t} w-\Delta w+b \partial_{z} w=-\lambda b \quad \text { in } G \text {. }
$$


Since $u=|\nabla u|=0$ on $\Phi$, also $w=0$ on $\Phi$ and if we show that

$$
w \leqslant m \text { on } L,
$$

then by the maximum principle one has $w-m \zeta \leqslant 0$ in $G$ and (7.2) will be proved.

Next let us recall that by the assumptions

$$
u(t) \underset{t \rightarrow \infty}{\rightarrow} u_{\infty} \text { in } C^{1, \alpha}\left(\bar{\Omega}^{\prime}\right), \quad \forall 0 \leqslant \alpha<1,
$$

and since $u,|\nabla u| \in C^{0}(G \cup \partial G)$ one has

$$
\sup _{G} u+\sup _{G}|\nabla u| \leqslant k
$$

and

$$
\gamma(\varepsilon)=\inf \left\{\partial_{z} u(X, t):(X, t) \in G \text { and } u(X, t)>\varepsilon\right\}>0 .
$$

Indeed, if $\gamma(\varepsilon) \leqslant 0$ one finds a sequence $G \ni\left(X_{n}, t_{n}\right) \rightarrow\left(X_{0}, t_{0}\right) \in G \cup \partial G$ if the $t_{n}$ are bounded, such that $u\left(X_{n}, t_{n}\right) \geqslant \varepsilon$ and $\partial_{z} u\left(X_{n}, t_{n}\right) \rightarrow \gamma(\varepsilon) \leqslant 0$, hence $u\left(X_{0}, t_{0}\right) \geqslant \varepsilon$ and $\partial_{z} u\left(X_{0}, t_{0}\right)=\gamma(\varepsilon) \leqslant 0$ which is a contradiction of $\partial_{z} u>0$ whenever $u>0$; if $t_{n} \rightarrow \infty$ and $X_{n} \rightarrow X_{0}$, by (7.5) one gets $u_{\infty}\left(X_{0}\right) \geqslant \varepsilon$ and $\partial_{z} u_{\infty}\left(X_{0}\right)=\gamma(\varepsilon) \leqslant 0$ which is also impossible by the same reason (see Theorem 3.1).

Now we can show that (7.4) holds by letting

$$
\varepsilon_{0}=\frac{m}{1+2 k} \quad \text { and } \quad A_{0}=\frac{k}{\gamma\left(\varepsilon_{0}\right)} \quad(\text { see }(7.6) \text { and (7.7)), }
$$

because if $(X, t) \in L$ and $u(X, t) \leqslant \varepsilon_{0}$ one gets

$$
w \leqslant \varepsilon_{0}+2 \varepsilon_{0} k \leqslant m \quad\left(\text { since } \partial_{z} u \geqslant 0\right),
$$

or if $(X, t) \in L$ and $u(X, t)>\varepsilon_{0}$ one has

$$
w \leqslant k+2 \varepsilon_{0} k-A_{0} \gamma\left(\varepsilon_{0}\right) \leqslant m .
$$

Finally, (7.2) implies that the directional derivative $\eta \cdot \nabla u(t) \geqslant 0$ in $F$, where $\eta=$ $(\varepsilon, \varepsilon, A)$, with $|\varepsilon| \leqslant \varepsilon_{0}$ and $A \geqslant A_{0}$; therefore $F \cap\{\tau=t\}$ verifies a cone property uniformly in $t$, which proves (7.1).

Remark 7.1. These arguments are actually very similar to the proof of the local Lipschitz continuity of $\psi$ both in space and time, which is based on the local property $v+\delta \partial_{t} v+\varepsilon \partial_{x} v+\varepsilon \partial_{y} v-A \partial_{z} v \leqslant 0$ for $0<\delta \leqslant \delta_{0},|\varepsilon| \leqslant \varepsilon_{0}$ and $A \geqslant A_{0}$, where $\psi$ and $v$ are the auxiliary functions introduced in the proof of Theorem 5.1 (recall $\partial_{t} v \geqslant 0$ ).

TheOREM 7.1. Assume (7.1) and under the notation of Theorem 6.1, if $\mu(t) \rightarrow 0$ (resp. $\mu(t)=O\left(t^{-\alpha}\right)$ or $\left.\mu(t)=O\left(e^{-\alpha t}\right)\right)$, one has the asymptotic behavior for $t \rightarrow \infty$ :

$$
\phi(t) \rightarrow \phi_{\infty} \quad \text { in } C^{0, \nu}\left(\bar{\Gamma}^{\prime}\right) \quad(\text { for any } 0 \leqslant \nu<1)
$$

or

$$
\left\|\phi(t)-\phi_{\infty}\right\|_{C^{0, \nu}\left(\Gamma^{\prime}\right)}=O\left(t^{-\beta}\right) \quad\left(\operatorname{resp} . O\left(e^{-\gamma t}\right)\right)
$$

for any smooth $\Gamma^{\prime}, \bar{\Gamma}^{\prime} \subset \Gamma$, any $\alpha>0$ and some $\left.\beta=\beta\left(\nu, \Gamma^{\prime}\right) \in\right] 0, \alpha\left[\right.$ or $\gamma=\gamma\left(\nu, \Gamma^{\prime}\right)$ $\in] 0, \alpha[$. 
Proof. The results follow easily by interpolating (6.4) or $\left(6.4^{\prime}\right)$ with (7.1). Indeed for any function $f \in C^{0.1}\left(\bar{\Gamma}^{\prime}\right)$ one has the inequality of Gagliardo-Nirenberg [N] (recall $\left.\Gamma^{\prime} \subset \mathbf{R}^{2}\right)$ :

$$
\|f\|_{p} \leqslant C_{1}\|\nabla f\|_{\infty}^{\sigma}\|f\|_{1}^{1-\sigma}+C_{2}\|f\|_{1}, \quad \forall \sigma \in[0,1] \text { and } 1 / p=(1-\sigma)-\sigma / 2,(7.9)
$$

where, if $1 / p \in[0,1],\|\cdot\|_{p}$ stands for the $L^{p}\left(\Gamma^{\prime}\right)$-norm and, if $1 / p \in[-1 / 2,0[$ for the Hölder semi-norm $[f]_{\nu}=\sup _{X \neq Y}\left[|f(X)-f(Y)| /|X-Y|^{\nu}\right]$ with $\nu=-2 / p$, and where $C_{1}, C_{2}$ are positive constants depending only on $\Gamma^{\prime}$. Using Young's inequality with $\varepsilon>0$ one finds

$$
\|f\|_{p} \leqslant\left(C_{2}+C_{1}\left(\frac{1-\nu}{3}\right) \varepsilon^{-3 /(1-\nu)}\right)\|f\|_{1}+C_{1}\left(\frac{2+\nu}{3}\right) \varepsilon^{3 /(\nu+2)}\|\nabla f\|_{\infty}
$$

where $p=-2 / \nu$ for $0<\nu<1$ and $p=\infty$ for $\nu=0$.

Now take $f=\phi(t)-\phi_{\infty}$ and choose $\varepsilon$ appropriately, according to the cases (6.4) or $\left(6.4^{\prime}\right)$, to complete the proof.

Acknowledgment. The author would like to thank Dietmar Kröner for useful conversations concerning Theorem 5.1, the Fundação Calouste Gulberkian for partial support and the Institute for Mathematics and its Applications at the University of Minnesota for hospitality while this research was concluded.

\section{REFERENCES}

[A] H. W. Alt, The fluid flow through porous media. Regularity of the free surface, Manuscripta Math. 21, 255-272 (1977)

[BC] C. Baiocchi and A. Capelo, Disequazioni variazionali e quasivariazionali. Applicazioni a problemi di frontieralibera, Quaderni dell' U.M.I., Pitagora, Bologna (1978) (See also English translation, John-Wiley and Sons, New York 1984)

[B] H. Brézis, Nouveaux théorèmes de régularité pour les problèmes unilatéraux, Rencontre entre Physicien Théoricien et Mathématicien, Strasbourg, Vol. 12 (1971)

[Br] T. Brière, Application des Méthodes Variationnelles à la cristallisation d' un métal par passage dans une gaine de refroidissement, Ann. Fac. Sci. Toulouse Math. 2, 219-247 (1980) (See also Thèse $3^{\text {ème }}$ cycle, Paris VI, 1976)

[C1] L. A. Caffarelli, The regularity of free boundaries in higher dimensions, Acta Math. 139, 155-184 (1978)

[C2] L. A. Caffarelli, A remark on the Hausdorff measure of a free boundary and the convergence of coincidence sets, Boll. Unione Math. Ital. 18, 1297-1299 (1981)

[CR] M. Chipot and J. F. Rodrigues, On the steady-state continuous casting Stefan problem with nonlinear cooling, Quart. Appl. Math. 40, 476-491 (1983)

[D] G. Duvaut, Résolution d'un problème de Stefan, C. R. Acad. Sc. Paris, 276A, 1461-1463 (1973)

[F] A. Friedman, Variational Principles and Free Boundary Problems, John Wiley and Sons, New York (1982)

[FK] A. Friedman and D. Kinderlehrer, A one-phase Stefan problem, Indiana Univ. Math. J. 24, 1005-1035 (1975)

[FT] A. Friedman and A. Torelli, A free boundary problem connected with nonsteady filtration in porous media, Nonlinear Analysis, Theory Meth. and Appl. 1, 503-545 (1977) (See correction in 2 (1978), 513-518)

[KS] D. Kinderlehrer and G. Stampacchia, An Introduction to Variational Inequalities and Their Applications, Academic Press, New York (1980)

[K] D. Kröner, The regularity of the free boundary for a nonsteady filtration problem in the case of a compressible flow, Preprint no. 661, SFB72, Universität Bonn (1984), (to appear in Boll. Un. Mat. Ital.)

[LS] O. A. Ladyzenskaya, V. A. Solonnikov and N. N. Ural'ceva, Linear and quasilinear equations of parabolic type, Amer. Math. Soc. Transl. Monog. 23 (1968)

[L1] J. L. Lions, Quelques méthodes de résolution des problèmes aux limites nonlinéaires; Dunod, Paris (1969)

[L2] J. L. Lions, Sur quelques questions d'Analyse, de Méchanique et de Contrôle Optimal, Presses Univ. Montréal (1976) 
[MS] M. K. V. Murthy and G. Stampacchia, A variational inequality with mixed boundary conditions, Israel J. Math. 13, 188-224 (1972)

[N] L. Nirenberg, An extended interpolation inequality, Ann. Scuola Norm. Sup. Pisa 20, 733-737 (1966)

[R1] J. F. Rodrigues, Sur un problème à frontière libre stationnaire traduisant la cristallisation d' un métal, C. R. Acad. Sc. Paris 290-A, 823-825 (1980) (See also Ph.D. Thesis, Lisbon, 1982)

[R2] J. F. Rodrigues, Stabilité de la frontière libre dans le problème de la digue, C. R. Acad. Sc. Paris 294-I, $565-568(1982)$

[R3] J. F. Rodrigues, Some remarks on the asymptotic behavior of strong solutions to monotone parabolic variational inequalities, Rend. Mat. (1984)

[R4] J. F. Rodrigues, Aspects of the variational approach to a continuous casting problem, Research Notes in Math. (Pitman) 120, 72-83 (1985)

[T1] A. Torelli, On a free boundary value problem connected with a nonsteady filtration phenomenon, Ann. Scuola Norm. Sup. Pisa 4, 33-59 (1977)

[T2] A. Torelli, Existence and uniqueness of the solution for a nonsteady free boundary problem, Boll. Unione Mat. Ital. (5) 14-B, 423-466 (1977)

[T3] A. Torelli, On a free boundary problem connected with a nonsteady filtration phenomenon of compressible fluid, Pub. no. 148, Lab Anal. Num. del C. N. R., Pavia (1977) 